\title{
A case of rectal villous adenoma in a female patient presenting with chronic diarrhea and acute renal
}

\section{failure}

\author{
Sheref A. Elseidy ${ }^{1 *}$, Haitham H. Alzamli ${ }^{1}$, Ahmed A. Abd Alkader ${ }^{2}$, Esraa Mamdouh ${ }^{1}$, Marina S. \\ Ghaly ${ }^{1}$ \\ 1Sheref A. Elseidy, Haitham H. Alzamli, Esraa Mamdouh, Marina S. Ghaly: Department of Internal medicine, Ain \\ Shams University, Cairo, Egypt \\ ${ }^{2}$ Abmed A. Abd Alkader: Department of rheumatology, Ain Shams University, Cairo, Egypt
}

\begin{abstract}
Secretory villous adenoma is one of the rare causes of severe secretory diarrhea, acute kidney injury and refractory electrolyte imbalance is the classic triad of this disorder named McKittrick-Wheelock syndrome. Aggressive hydroelectrolyte rebalancing is often needed, and curative treatment is obtained only with complete removal of the lesion, by endoscopic resection or surgery. High clinical suspicion in patients with severe dehydration due to secretory diarrhea and concomitant electrolyte imbalance is both mandatory and essential for early diagnosis and treatment before complications arise.

In this case we describe a female patient suffering from 6 months of secretory diarrhea, presented to our emergency department with severe dehydration, acute renal failure, hyponatremia and hypokalemia. After rehydration and electrolyte replacement, confirmation of the diagnosis was done by colonoscopy and biopsy showing 3 sessile colonic polyps ( 2 at distal end of Transverse colon, largest one at descending colon) the biopsy confirmed the presence of villous adenoma with high grade dysplasia. Normalization of the electrolyte imbalance and the kidney functions was done and she was successfully treated with surgical resection that lead to complete resolution of symptoms.

McKittrick-Wheelock syndrome is a rare life threatening depletion syndrome that can lead to acute kidney injury and electrolyte imbalance with concomitant organ damage. Early management by vigorous repletion therapy and curative surgical resection will mostly resolve the symptoms and complications.
\end{abstract}

Key Words: Villous adenoma, chronic diarrhea, electrolyte imbalance, acute renal failure

\section{Introduction}

First described in 1954, McKittrick-Wheelock syndrome a triad of and electrolyte depletion, Dehydration, mucous diarrhea cause by a secretory colorectal adenoma or adenoncarcinoma (1) presenting with symptoms of hyponatremia ranging from gastrointestinal upset up to seizures and coma, hypokalemia with fatique and ileus up to cardiac dysfunction and arrythmias and hypotension (2-5) current management strategies include prompt and aggressive fluid and electrolyte replacement till normalization of kidney functions and improvement of general condition thus preventing the often grave complications of this hydroelectrolytic imbalance on organs followed by immediate curative surgical resection or endoscopic resection of the colorectal mass depending on the characteristics of the mass that include size, site and accessibility $(7,8)$.

\section{Case Report}

Our study reports a female patient presented to our ER with severe weakness, tachycardia and a 6 month history of nonbloody mucoid diarrhea with 5-6 motions/d, not improved with fasting, several courses of antibiotics.

There was significant un-estimated weight loss; along with mild epigastric pain.

Examination showed: pallor, hypotension, HR: 120, bl.pr: $80 / 40$

Labs showed: BUN: 84, creatinine: 1.1, HCT: 63, k: 1.8, Na: 109 and TLC: 9.1

ESR was found to be 30 and CRP $<6$ with negative tumor markers with values: AFP: 3.1, CEA: 1.1, CA 19-9: 5.2 and CA 125: 9.7

Extensive volume repletion was done after admission with ringer and potassium infusion 
which lead to resolution of both clinical signs and symptoms, elevation of blood pressure and resolution of tachycardia with improvement of serum sodium and potassium with follow up results of (Na: 128 then 135, K: 3.1, 3.3, 4.3).

PAUS: Liver of average size, homogenous \& with smooth borders and $2 \mathrm{~cm}$ gall bladder stone

CT Abdomen \& Pelvis: Hepatomegaly $17 \mathrm{~cm}$ (Homogenous CT density, no HFLs), Single GB stone (2cm, normal GB wall thickness) and Multiple uterine fibroids.

Colonoscopy was done and revealed 3 sessile colonic polyps (2 at distal end of Transverse colon, largest one at descending colon) with apparently normal mucosa in-between. Multiple biopsies were taken. Microscopic picture: Polypoid colonic mucosa showing villoglandular/glandular architecture with marked nuclear stratification of covering epithelium and the diagnosis of villous adenoma with high grade dysplasia was achieved.

Volume and electrolyte repletion was continued till surgical intervention. After clinical and laboratory improvement the patient underwent endoscopic excision of the polyps which alleviated her symptoms.

\section{Discussion}

Electrolyte depletion syndrome is a rare disorder caused by fluid and electrolyte hypersecretion from a villous adenoma. Dehydration with severe hyponatremia, hypokalemia, metabolic acidosis, acute renal failure and watery diarrhea is typical. Exact diagnosis is difficult and requires a high level of suspicion and early colonoscopy for visualization and biopsy. Most cases present at late stages mostly due to being misdiagnosed as infectious diarrhea as in this case. Villous adenomas rarely cause a secretory diarrhea syndrome and only about $3 \%$ of villous adenomas have secretory activity (9), The tumor is usually located at the rectosigmoid or rectum and often is $3-4 \mathrm{~cm}$ or more in diameter $(9,10)$, also secretory villous adenomas associated with depletion syndrome are large, ranging from 7 to $18 \mathrm{~cm}$ with increased diarrheal volumes of $350-3000 \mathrm{~mL}$ that leads to hypovolemia, metabolic imbalances and acute renal failure. The two main determinants of whether the adenoma would produce depletion syndrome are the large size which provides higher surface area for secretion, and the distal location which spares only a small area of normal mucosa remains for fluid absorption (11). Complications are grave and can be prevented by adequate and prompt rehydration and acid base correction is not immediately performed and patient is stabilized (7-8).

Complications range from dehydration, asthenia and tachycardia up to profound acute renal injury and myocardial affection in the form of fatal arrhythmias (1-2). it was found that up to $80 \%$ of these villous adenomas have been reported to host foci of adenocarcinoma, making complete surgical excision of the lesion as the most appropriate treatment to decrease the chance of cancer and to resolve the depletion syndrome. Also surgical polypectomy is advised if the polyp is larger than $3 \mathrm{~cm}$ or sessile as villous adenomas often are (12).

A triad of acute prerenal failure, electrolyte disturbances and chronic diarrhea combined with the absence of other major causes of prolonged diarrhea should raise the indication for an early complete colonoscopy for prompt diagnosis in typical cases and to intervene with an appropriate therapy, resolving a condition that may develop life-threatening complications and that, if left untreated for a long time, could evolve in invasive cancer. (2).

\section{References}

1. McKittrick LS, Wheelock FC. Carcinoma of the colon. 1954. Dis Colon Rectum 1997; 40: 14941495.

2. Choi WH, Ryuk J, Kim HJ, et al. A case of giant rectal villous tumor with severe fluid-electrolyte imbalance treated by laparoscopic low anterior resection. J Korean Surg Soc 2012; 82: 325-329.

3. McCabe RE, Kane KK, Zintel HA, Pierson RN. Adenocarcinoma of the colon associated with severe hypokalemia: report of a case. Ann Surg 1970; 172: 970-974

4. Older J, Older P, Colker J, Brown R. Secretory villous adenomas that cause depletion syndrome. Arch Intern Med 1999; 159: 879-880.

5. Targarona EM, Hernandez PM, Balague C, et al. McKittrick-Wheelock syndrome treated by laparoscopy: report of 3 cases. Surg Laparosc Endosc Percutan Tech 2008; 18: 536-538.

6. Konishi S, Nakada I, Satani T, Kasuga T, Watanabe Y, Tabuchi T. Villous rectal adenoma as a rare cause of acute renal failure (McKittrick-Wheelock syndrome) Coloproctology 2010; 30: 251-253.

7. Popescu A, Orban-Schiopu AM, Becheanu G, Diculescu M. McKittrick-Wheelock syndrome-a rare cause of acute renal failure. Rom J Gastroenterol 2005; 14: 63-66.

8. Emrich J, Niemeyer C. The secreting villous adenoma as a rare cause of acute renal failure. Med Klin (Munich) 2002; 97: 619-623.

9. Watari J, Sakurai J, Morita T, et al. A case of Cronkhite-Canada syndrome complicated by McKittrick-Wheelock syndrome associated with 
advanced villous adenocarcinoma. Gastrointest Endosc 2011; 73: 624-626.

10. Older J, Older P, Colker J, et al. Secretory villous adenomas that cause depletion syndrome. Arch Intern Med 1999; 159: 879-880.

11. Lee YS, Lin HJ, Chen KT. McKittrick-Wheelock syndrome: a rare cause of life-threatening electrolyte disturbances and volume depletion. J Emerg Med 2012; 43: 171-173.

12. Annamaria Agnes, Domenico Novelli, Giovanni Battista Doglietto and Valerio Papa. A case report of a giant rectal adenoma causing secretory diarrhea and acute renal failure: McKittrickWheelock syndrome. BMC Surgery 2016; 16: 39. 